\title{
Aplicación del Método de los Elementos Finitos para la Simulación de las Transmisiones por Flanco en Uniones con Suelos Flotantes
}

\author{
Romina Del Rey ${ }^{(1)}$, Jesús Alba ${ }^{(1)}$, Jaime Ramis ${ }^{(2)}$ y Eva Escuder ${ }^{(1)}$ \\ (1) Universidad Politécnica de Valencia, Instituto para la Gestión Integral de Zonas Costeras, \\ Esc. politécnica superior de Gandía, C/ Paraninfo n¹ 46730 Grao de Gandía-Valencia (España) \\ (e-mail: roderey@doctor.upv.es, jesalba@fis.upv.es, evescude@fis.upv.es) \\ (2) Universidad de Alicante, Dpto. Física, Ingeniería de Sistemas y Teoría de la Señal, \\ Apdo. Correos, 99; 03080 Alicante-España (e-mail: jramis@ua.es)
}

Recibido Dic. 17, 2009; Aceptado Feb. 10, 2010; Versión Final recibida Mar. 23, 2010

\begin{abstract}
Resumen
En este trabajo se plantea el uso del método de los elementos finitos para el estudio de las transmisiones laterales a través del índice de reducción de vibraciones, para el caso de soluciones constructivas con suelos flotantes. Por una parte, la inserción de láminas elásticas en nuevas soluciones constructivas es en la actualidad una práctica muy común. Por otra parte, los procedimientos de ensayo para el estudio acústico de este conjunto (forjado con lámina elástica) son muy laboriosos y las ecuaciones empíricas existentes no satisfacen la realidad en muchos de los casos. Es por este motivo que se plantea el estudio mediante los elementos finitos, lo que nos permitirá dar solución numérica a un gran conjunto de diferentes soluciones constructivas y poder así mejorar las predicciones numéricas para el aislamiento acústico.
\end{abstract}

\section{Application of the Finite Elements Method for the Simulation of Flanking Transmissions in Joints with Floating Floors}

\begin{abstract}
This paper presents the use of the finite elements method to study the flanking transmission through the use of the vibration reduction index, for the case of constructive solutions that use floating floors. On one side, the insertion of elastic interlayer in new building solutions is now a very common practice. On the other side, the test procedures for the acoustic study of this kind of sets (concrete floor with elastic interlayer) are very laborious and the existing empirical equations do not match reality in many cases. For this reason, the study of the problem using finite elements would allows giving numerical solution to a wide range of different constructive solutions and numerical prediction for the acoustical isolation could be improved.
\end{abstract}

Keywords: flanking transmission, sound insulation, vibracional reduction index, acoustic prediction, finite elements. 


\section{INTRODUCCIÓN}

La instalación de suelos flotantes para reducir el ruido de impacto es una de las mejoras importantes en las soluciones constructivas de la edificación europea. Estos suelos además son obligatorios en países como España, con la entrada en vigor de forma obligatoria en abril de 2009 del Documento Básico de Protección Frente al Ruido del Código Técnico de la Edificación, (DB-HR del CTE, 2007). Con estos suelos flotantes se pretende, además de reducir los niveles del ruido de impacto, disminuir la transmisión del ruido a través de los flancos o elementos laterales.

Las normas europeas CEN EN 12354-1(2000) y CEN EN 12354-2 (2000) plantean métodos de predicción para la estimación del aislamiento acústico a ruido aéreo y ruido de impacto a través de los elementos que componen la solución constructiva. En estas normativas se valora la transmisión del ruido a través de los flancos laterales mediante el índice de reducción vibracional. Este parámetro se puede obtener mediante el uso de fórmulas empíricas reconocidas en las normas citadas para algunas soluciones constructivas y en un conjunto determinado y limitado de diseños rígidos o con elementos elásticos insertados. En el caso de inserción de elementos elásticos, aparecen soluciones limitadas con elementos con una masa por unidad de área elevada a los que se les ha colocado una banda elástica determinada sin contemplar otros casos.

Por otro lado, para suelos flotantes, no produce ninguna corrección respecto a los valores de uniones rígidas en el valor del índice de reducción vibracional, asumiendo que se produce una mejora directa en el suelo añadido sobre el forjado. Por tanto, las normas citadas plantean carencias en la obtención de las simulaciones, por falta de formulación para los casos con elementos elásticos y láminas flotantes.

Otra técnica que puede ser factible es la obtención de información del flanco en una edificación, mediante procedimientos de medida similares a los detallados en las normas ISO 10848 (2007), con el uso de diferente equipamiento y la medición de multitud de datos de diferencias de niveles de velocidad y tiempos de reverberación estructural, para obtener datos sobre transmisión de energía y pérdidas. En Alba et Al. , 2007 a, se muestra un estudio donde la misma solución constructiva para aislamiento acústico, tiene diferentes resultados de medición, debido a las diferentes condiciones de los flancos laterales. Se pueden encontrar diferentes trabajos sobre estudios de las transmisiones laterales, como son Craik y Osipov, 1995, Hopkins, 1999, Crispin al., 2006 y Alba et al., 2008. En estos trabajos y en las normas ISO 10848 (2007) se puede comprobar lo complejo de estos ensayos, que en algunos casos pueden durar varios días. Por tanto, el uso de herramientas numéricas es una buena alternativa.

En este trabajo se ha simulado el procedimiento de medición descrito en las normas ISO 10848 (2007), para obtener el índice de reducción de vibraciones, mediante el método de los elementos finitos. Han sido estudiadas diferentes configuraciones de uniones en forma de cruz $(+)$ y de té $(T)$, comparando las mismas situaciones sin y con lámina elástica que cumpla, en la medida de lo posible, las características que se señalan en la CEN EN 12354-1(2000).

\section{ÍNDICE DE REDUCCIÓN VIBRACIONAL}

El índice de reducción vibracional, $\mathrm{K}_{\mathrm{ij}}$, se define como la magnitud relacionada con la transmisión de potencia vibratoria a través de una unión entre elementos estructurales, normalizada con el objeto de hacerla una magnitud invariante. Se determina normalizando la diferencia de niveles de velocidad promediados en todas direcciones sobre la unión, con la longitud de la unión y la longitud de absorción equivalente, si fuera relevante, de ambos elementos según la siguiente ecuación:

$$
K_{i j}=\frac{D_{v, i j}+D_{v, j i}}{2}+10 \log \frac{l_{i j}}{\sqrt{a_{i} \cdot a_{j}}}
$$

En la expresión (1) $D_{v, i j}(d B)$ es la diferencia del nivel de velocidad entre los elementos i y j, cuando el elemento i es excitado, $D_{v, j i}(d B)$ es la diferencia del nivel de velocidad entre los elementos $j$ e $i$, 
cuando el elemento j es excitado, $\mathrm{l}_{\mathrm{ij}}(\mathrm{m})$ es la longitud común de la unión entre los elementos i y j, $\mathrm{a}_{\mathrm{i}}$ (m) y $a_{j}(m)$ son la longitud de absorción equivalente del elemento i o j respectivamente, que se puede calcular mediante la siguiente expresión:

$a_{i}=\frac{2.2 \cdot \pi^{2} \cdot S_{i}}{c_{0} \cdot T_{s, i}} \sqrt{\frac{f_{r e f}}{f}}$

Siendo $T_{s}(\mathrm{~s})$ el tiempo de reverberación estructural de cada elemento, $S\left(\mathrm{~m}^{2}\right)$ el área de cada elemento, $f(\mathrm{~Hz})$ la frecuencia central de la banda, $f_{\text {ref }}$ es la frecuencia de referencia, $1000 \mathrm{~Hz}$ y $\mathrm{c}_{0}(\mathrm{~m} / \mathrm{s})$ es la velocidad del sonido en el aire.

\section{Determinación experimental del índice de reducción vibracional}

Tal y como se especifica en ISO 10848, es posible obtener el valor de $\mathrm{K}_{\mathrm{ij}}$ utilizando como base las ecuaciones (1) y (2), a partir de la medición de la diferencia de niveles de velocidad de la unión en ambas direcciones y del tiempo de reverberación estructural de los dos elementos. En esta norma se detalla un procedimiento de ensayo que, posteriormente, será reproducido mediante simulación para obtener información del índice de reducción de vibraciones por el método de los elementos finitos. En este procedimiento se especifica que debe excitarse estructuralmente cada elemento en tres posiciones. Las posiciones de medida deben distribuirse aleatoriamente y no simétricamente. Además, la posición del transductor y de los puntos de excitación deben cumplir las siguientes distancias mínimas: $0.5 \mathrm{~m}$ entre los puntos de excitación y los límites del elemento a ensayar, $1 \mathrm{~m}$ entre los puntos de excitación y las posiciones de transductor asociadas y 0.5 m entre cada posición del transductor.

Para la medición del tiempo de reverberación estructural se puede seguir la normativa ISO 3382-2 (2008). Se excita el elemento constructivo con un golpe de martillo y se obtiene el tiempo que tarda en caer el sonido $60 \mathrm{~dB}$. La norma indica al menos tres puntos de excitación por, al menos, tres posiciones de transductor para la obtención del tiempo de reverberación estructural. Las condiciones de colocación son similares a las de la medida de la diferencia de velocidades.

La determinación de la diferencia de niveles de velocidad junto con el tiempo de reverberación estructural es el primer paso para la obtención de $\mathrm{K}_{\mathrm{ij}}$. Las expresiones (1) y (2) se basan en una serie de premisas sobre los campos acústicos de los elementos excitados, como la necesidad de campos difusos y acoplamiento débil entre los elementos que conforman la unión (CEN 12354-1, 2000). Esta condición de acoplamiento se refleja en la siguiente desigualdad:

$D_{v, i j} \geq 3-\lg \left(\frac{m_{i} f_{c j}}{m_{j} f_{c i}}\right) d B$

Donde $\mathrm{m}_{\mathrm{i}}\left(\mathrm{kg} / \mathrm{m}^{2}\right)$ y $\mathrm{m}_{\mathrm{j}}\left(\mathrm{kg} / \mathrm{m}^{2}\right)$ son las masas por unidad de área de cada elemento, y $\mathrm{f}_{\mathrm{ci}}(\mathrm{Hz})$ y $\mathrm{f}_{\mathrm{cj}}(\mathrm{Hz})$ son las frecuencias críticas de los elementos, que se pueden obtener de la siguiente relación:

$f_{c i}=\frac{c_{o}^{2}}{2 \pi} \sqrt{\frac{m_{i}}{B_{i}}}$

Siendo $\mathrm{B}_{\mathrm{i}}(\mathrm{Nm})$ la rigidez a flexión del elemento.

Si no se cumple la condición (3) el índice de reducción de vibraciones que se obtienen tal y como se indica en (1) no es representativo de la transmisión de energía entre los elementos sometidos a estudio. 
Fórmulas empíricas para el índice de reducción vibracional

En las normas CEN EN 12354-1(2000) y CEN EN 12354-2 (2000) se puede encontrar un anexo con expresiones para obtener el índice de reducción vibracional. Estas expresiones han sido obtenidas a través de datos empíricos, para tipos habituales de uniones. Tan solo existen expresiones empíricas para el caso de uniones en que los elementos a ambos lados de la unión en el mismo plano tienen la misma masa. La figura 1 muestra el caso de uniones rígidas.

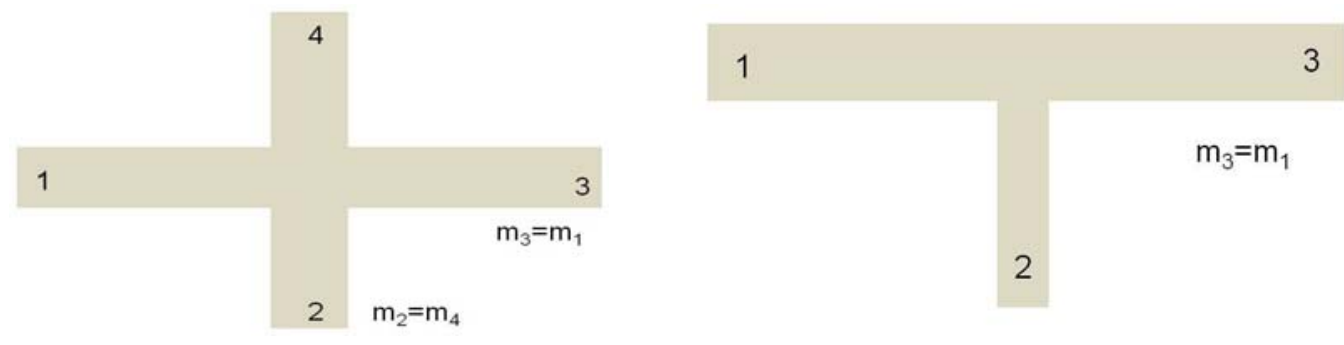

Fig. 1: Uniones rígidas a comparar. Unión de elementos homogéneos, en + (derecha) y en $\mathrm{T}$ (izquierda)

En el caso de la unión en cruz, se definen las siguientes relaciones para los índices de reducción vibracional asociados:

$K_{13}=8,7+17,1 \mathrm{M}+5,7 \cdot \mathrm{M}^{2} \quad \mathrm{~dB} ;$
$K_{12}=8,7+5,7 \cdot \mathrm{M}^{2}\left(=\mathrm{K}_{23}\right) \quad \mathrm{dB} ; \quad 0 \mathrm{~dB} /$ octava

En el caso de la unión en $\mathrm{T}$, se definen las siguientes relaciones para los índices de reducción vibracional asociados:

$$
\begin{aligned}
& K_{13}=5,7+14,1 \mathrm{M}+5,7 \cdot \mathrm{M}^{2} \quad \mathrm{~dB} ; \quad 0 \mathrm{~dB} / \text { octava } \\
& K_{12}=5,7+5,7 \cdot \mathrm{M}^{2}\left(=\mathrm{K}_{23}\right) \quad \mathrm{dB} ; \quad 0 \mathrm{~dB} / \text { octava }
\end{aligned}
$$

Las expresiones (5) y (6) están dadas en función de la magnitud M definida como:

$M=\lg \frac{m_{\perp i}^{\prime}}{m_{i}^{\prime}}$

Siendo $m_{i}^{\prime}$ la masa por unidad de superficie del elemento i en el camino de transmisión ij y m'」i la masa por unidad de superficie del otro elemento, perpendicular al i, que forma la unión. En el cálculo de estas masas sólo se cuenta el material base o forjado conectado a los elementos constructivos colindantes. Se puede observar como las magnitudes dadas por (5) y (6) no tienen dependencia con la frecuencia. De hecho, según se indica en el DB-HR, en general la transmisión es poco dependiente de la frecuencia desde 125 a $2 \mathrm{kHz}$, por lo que se considera $0 \mathrm{~dB} /$ octava.

En el caso de interponer elementos flexibles (figura 2), las fórmulas reconocidas son las siguientes:

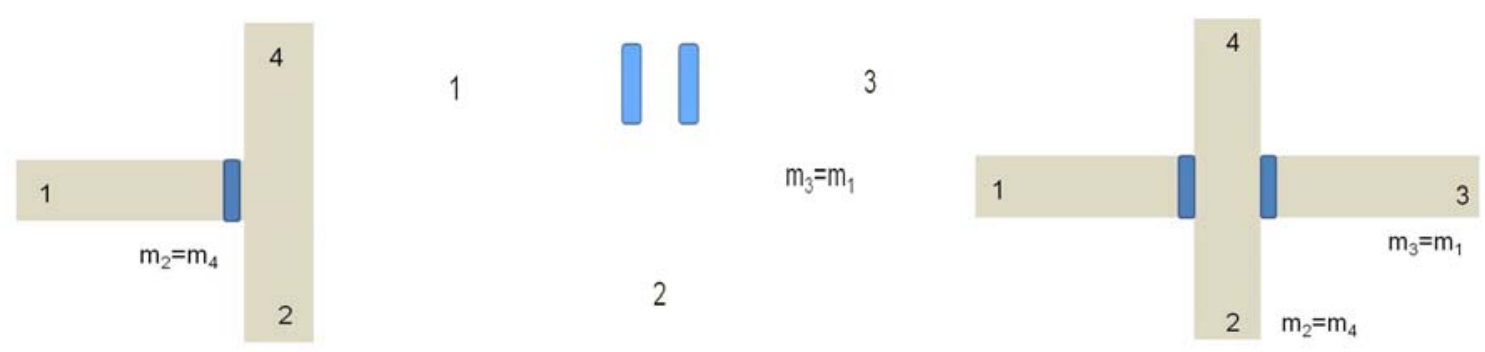

Fig. 2: Uniones con elementos flexibles insertados 
$K_{13}=5,7+14,1 \mathrm{M}+5,7 \cdot \mathrm{M}^{2}+2 \cdot \Delta_{1} \mathrm{~dB} ;$

$K_{24}=3,7+14,1 \mathrm{M}+5,7 \cdot \mathrm{M}^{2} \quad \mathrm{~dB} ; \quad-4 \mathrm{~dB} \leq \mathrm{K}_{24} \leq 0 \mathrm{~dB}$;

$K_{12}=5,7+5,7 \cdot \mathrm{M}^{2}+\Delta_{1}\left(=\mathrm{K}_{23}\right) \mathrm{dB}$;

donde:

$\Delta_{1}=10 \cdot \lg \left(\mathrm{f} / \mathrm{f}_{1}\right) \quad \mathrm{dB} \quad$ para $\mathrm{f}>\mathrm{f}_{1}$

$f_{1}=125 \mathrm{~Hz}$ si $\left(\mathrm{E}_{1} / \mathrm{e}_{1}\right) \approx 100 \mathrm{M} \mathrm{N} / \mathrm{m}^{3}$

Siendo $E_{1}\left(N / m^{2}\right)$ el módulo de Young de la lámina y $e_{1}(m)$ su espesor.

Las ecuaciones (8a), (8b) y (8c) incorporan un factor corrector $\Delta_{1}$ que representa la evolución en función de la frecuencia de $\mathrm{K}_{\mathrm{ij}}$. Las fórmulas de la norma son sólo válidas para una relación determinada entre el Módulo de Young y el espesor de la lámina interpuesta. Además, $\mathrm{f}_{1}=125 \mathrm{~Hz}$, se cumple sólo en el caso de ciertos suelos flotantes con una carga de mortero sobre la lámina.

\section{MODELO EN ELEMENTOS FINITOS}

Mediante el método de los elementos finitos, un sistema continuo se divide en una serie de porciones finitas delimitadas por nodos, cuyos grados de libertad constituyen las incógnitas del problema. La exactitud de los resultados depende del número de elementos utilizados en el mallado. En este caso se ha mallado con elementos planos de ocho nodos, con dos grados de libertad por nodo, con lo cual existen desplazamientos en los ejes $\mathrm{X}$ e $\mathrm{Y}$. El número medio de elementos por modelo de unión es de 10000. La ecuación del movimiento armónico a resolver es la siguiente:

$\{F(t)\}=[M]\left\{\frac{d^{2} y}{d t^{2}}\right\}+[C]\left\{\frac{d y}{d t}\right\}+[K]\{y\}$

Donde $\{\mathrm{F}(\mathrm{t})\}$ es la fuerza de excitación armónica, [M] es la matriz de masa, [C] es la matriz de amortiguamiento y $[\mathrm{K}]$ es la matriz de rigidez. En el trabajo de Zienkiewick, 1993, se describe la técnica. El tipo de solucionador utilizado ha sido Damped Method, ya que en los sistemas estudiados el amortiguamiento no puede ser ignorado. Se ha realizado la simulación sin interfase entre los elementos rígidos y el aire y las condiciones de contorno han sido de apoyos restringidos, esto es, empotramiento perfecto en las uniones.

\section{Modelos 2D de unión en cruz y en $T$}

Para realizar un acercamiento al problema, se ha simulado en ANSYS las condiciones de la normas ISO 10848 descritas anteriormente, respecto a la colocación de fuentes de excitación y transductores de medida y respecto al tamaño de las muestras, Se han simulado materiales de $2400 \mathrm{~kg} / \mathrm{m}^{3} \mathrm{de}$ densidad, $32 \cdot 10^{9} \mathrm{~Pa}$ de módulo de Young y 0,22 de coeficiente de Poisson, equivalentes al hormigón armado. Los espesores simulados son de 10, 20 y $30 \mathrm{~cm}$ y el factor de pérdidas interno de 0,01. Respecto a la lámina elástica, se ha escogido una que cumpla con la condición impuesta con la fórmula (9b), respecto a la relación entre el módulo de Young y su espesor, y un factor de pérdidas de 0,2 .

De esta forma, como fuerza de excitación se aplica una fuerza constante en el margen de frecuencias considerado de $1 \mathrm{~N}$ en las posiciones marcadas con las flechas (ver figura 3 ) y se obtiene, mediante la simulación en elementos finitos en ANSYS, las velocidades en puntos marcados con estrellas. Entre distintas posiciones de fuerza se ha dejado $1 \mathrm{~m}$ y entre puntos de medida, 0,5 $\mathrm{m}$, tal y como indica la norma. En la figura 3 se pueden observar dos esquemas de configuración en T, con los puntos de excitación (flechas) y puntos de registro de velocidad (estrellas) tal y como indica la normativa $(10848,2007)$. 

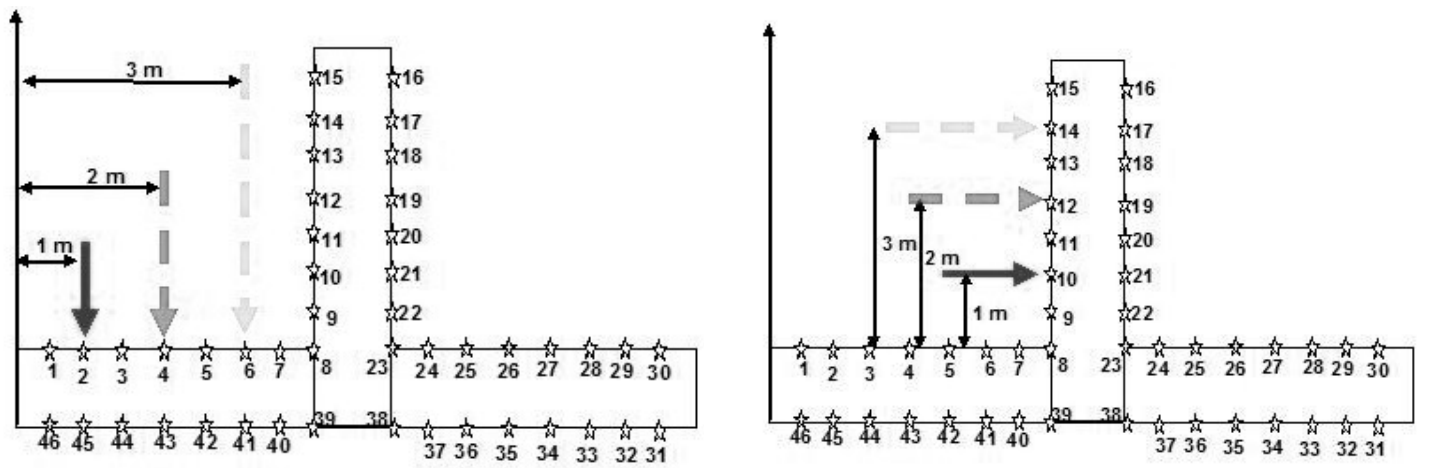

Fig. 3: Modelos para unión rígida en T de elementos homogéneos

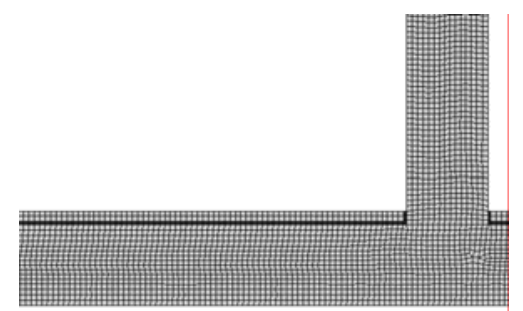

Fig. 4: Detalle de malla en T con lámina elástica

A partir de las simulaciones, se obtiene el valor de las velocidades en diferentes posiciones i y j. A partir de estos datos, se obtiene el promedio según la norma y se obtiene la media de diferencia de niveles de velocidad.

Para la obtención del tiempo de reverberación estructural, se sigue la siguiente expresión, dada por la CEN EN 12354-1(2000):

$T_{s}=\frac{2,2}{f \eta_{T}}$

Donde $\eta_{\mathrm{T}}$ es el factor de pérdidas total que se puede obtener mediante la relación:

$\eta_{T}=\eta_{\text {int }}+\frac{m}{485 \sqrt{f}}$

Siendo m la masa por unidad de área del elemento. La fórmula es válida, según la CEN EN 12354-1 para masas por unidad de área menores de $800 \mathrm{~kg} / \mathrm{m}^{2}$.

\section{RESULTADOS}

\section{Resultados de unión en cruz}

En primer lugar se presentan resultados del índice de reducción vibracional en función de la frecuencia, desde 200 a $1250 \mathrm{~Hz}$, y valores globales como media aritmética, tal y como indica la normativa. K12R representa la transmisión lateral desde la parte horizontal izquierda a la cara vertical y K13 la transmisión desde la parte horizontal izquierda a la derecha, siguiendo la secuencia de números marcada en la figura 2. En la figura 5 se presentan resultados de la diferencia de niveles de velocidad promediadas para diferentes posiciones de excitación (C2: excitación a 2 metros del extremo del elemento y a 2 metros de la unión, C3: excitación a 3 metros del extremo del elemento y a 1 metro de la unión) y teniendo en cuenta el límite marcado por la ecuación (3) (línea continua). Se compara el caso de relación de masas 0,5, con lo que $\mathrm{M}<0$. 

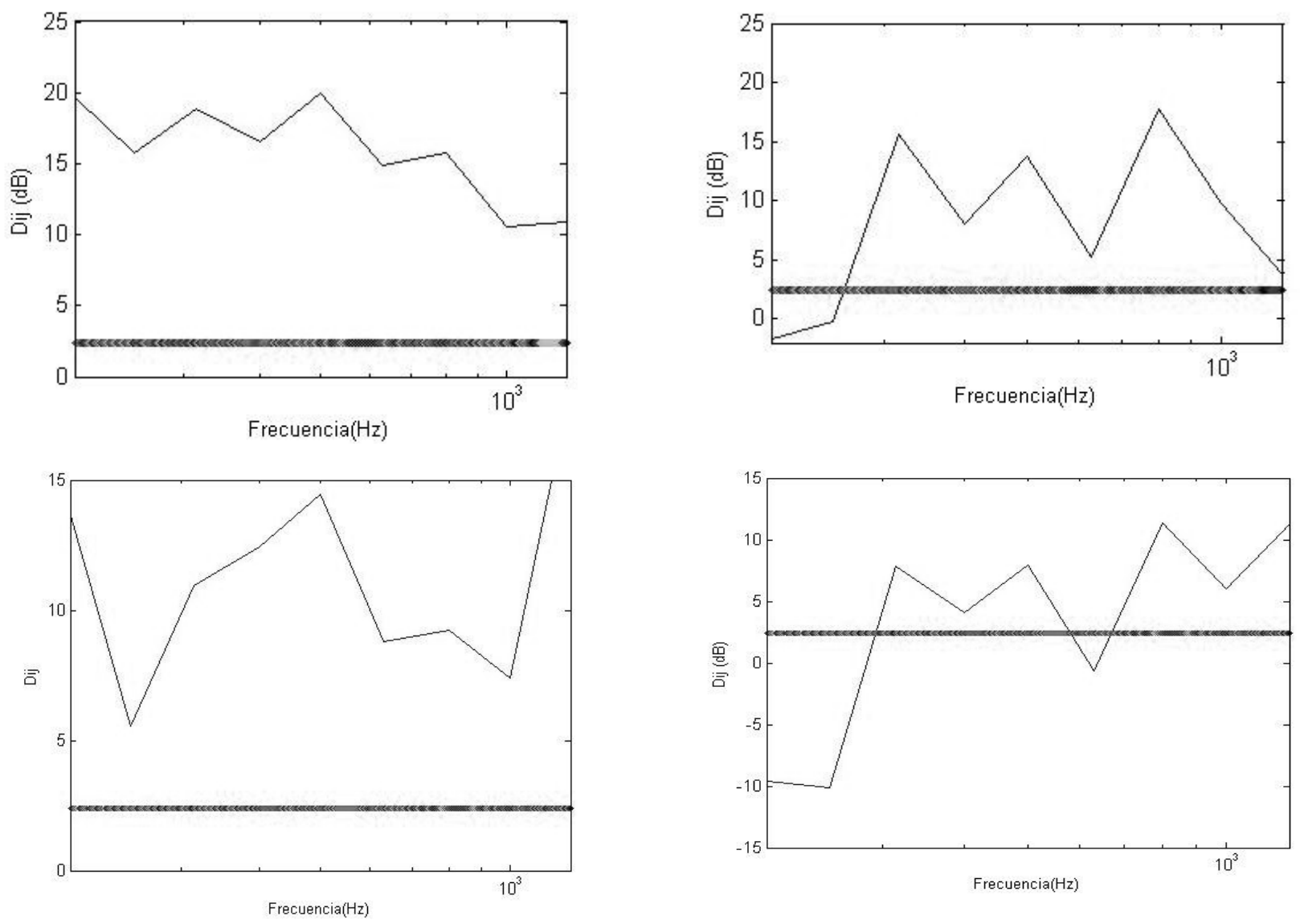

Fig. 5: Diferencia de niveles de velocidades para K12R (superiores) y K13 (inferiores) en el caso de $\mathrm{M}<0$ y uniones rígidas, para C2 (izquierda) y C3 (derecha).

En las figuras 6 se presentan los valores dependientes de la frecuencia de los casos estudiados de la unión rígida, para $\mathrm{M}=0$. Se presentan resultados para $10 \mathrm{~cm}$ de espesor, correspondiente a $240 \mathrm{~kg} / \mathrm{m}^{2}, 20 \mathrm{~cm}\left(480 \mathrm{~kg} / \mathrm{m}^{2}\right)$ y $30 \mathrm{~cm}\left(720 \mathrm{~kg} / \mathrm{m}^{2}\right)$. Según las expresiones (5), en este caso, el índice de reducción de vibraciones debería ser independiente de la frecuencia y su valor de 8,7 dB. Autores como Craik (2001) justifican desviaciones entorno a los 2 dB, cuando se siguen protocolos de medición, debido a acoplamiento entre los diferentes elementos estructurales cuando éstos son excitados para poder obtener la diferencia de niveles de velocidad. Otros autores como Pedersen (1995) estudiando uniones rígidas obtienen valores cercanos a los empíricos con las mediciones, con diferencias entorno 1,5 dB.

K12R. M=0. Rigido

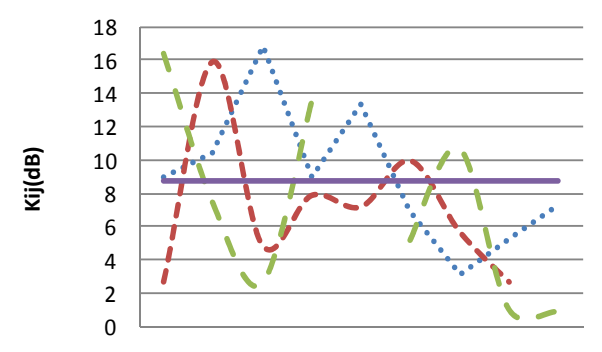

20025031540050063080010001250

$f(\mathrm{~Hz})$

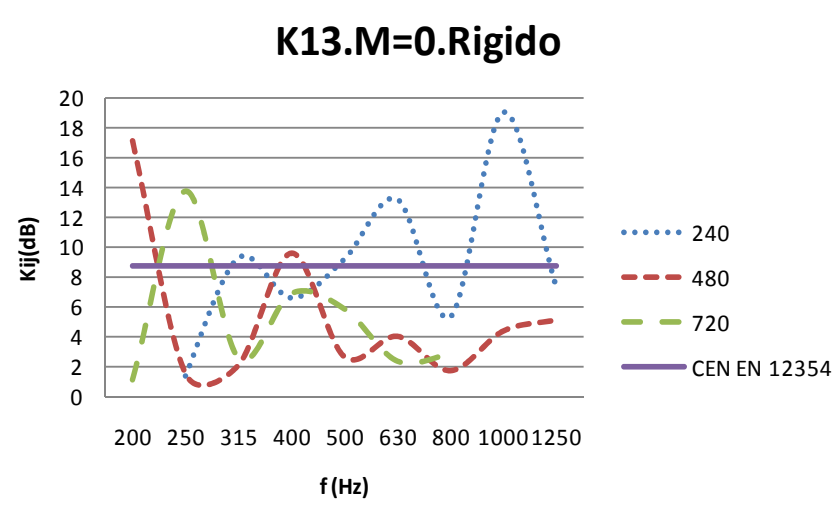

$f(\mathrm{~Hz})$

Fig. 6: Valores del índice de reducción vibracional (K12R y K13) para unión rígida y M=0.

Los resultados obtenidos en el caso de suelo flotante se muestran en la figura 7. Se comparan los resultados obtenidos con los valores según por la norma CEN EN 12354-1(2000). 

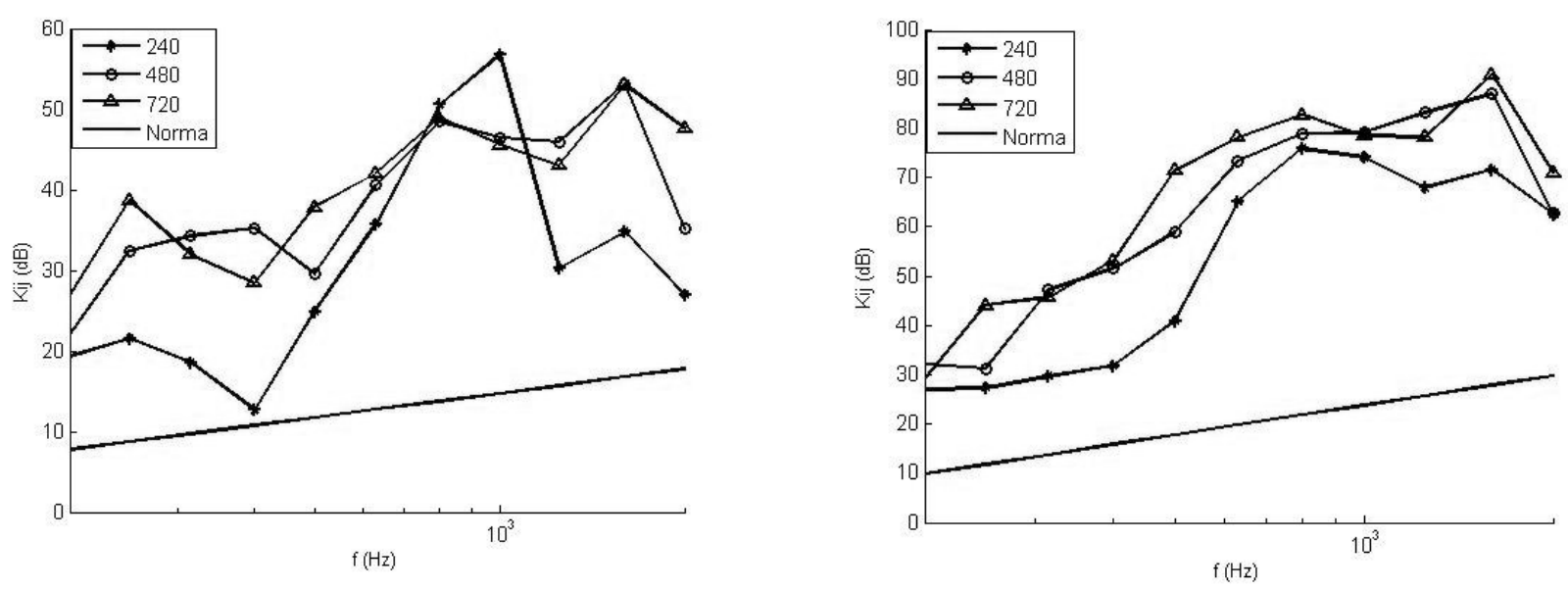

Fig. 7: Valores del índice de reducción vibracional K12R (izquierda) y K13 (derecha) en suelo flotante para $\mathrm{M}=0$.

Tal y como se puede observar en las fórmulas (8) y (9), el paso de una unión rígida a una con elemento elástico insertado se corrige con el parámetro $\Delta$. Esta variable depende de la frecuencia y de la frecuencia de resonancia propia del sistema masa-muelle conformado por una banda elástica y la masa del elemento de unión, aunque la norma CEN-EN 12354-1 sólo ofrece un caso en particular. Según este resultado y tomando como base Pedersen (2001) se propone un ajuste de los resultados de la figura 7 , donde $M=0$, según la expresión $K_{i j}=a+b \log f$. Este ajuste se muestra en la figura 8. Se puede observar cómo el coeficiente b es cercano a 20, siguiendo el mismo patrón que un gran número de resultados que observó Pedersen (2001) al estudiar las pérdidas por transmisión cuando en el camino es necesario el paso por dos láminas flexibles.

\section{Resultados de unión en T}

Se han obtenido resultados del índice de reducción vibracional en los mismos caminos anteriores para uniones en T. En la figura 9 se presentan los valores dependientes de la frecuencia de los casos estudiados de la unión rígida en $\mathrm{T}$ para $\mathrm{M}=0$. Se presentan resultados para $10 \mathrm{~cm}$ de espesor, correspondiente a $240 \mathrm{~kg} / \mathrm{m}^{2}, 20 \mathrm{~cm}\left(480 \mathrm{~kg} / \mathrm{m}^{2}\right)$ y $30 \mathrm{~cm}\left(720 \mathrm{~kg} / \mathrm{m}^{2}\right)$. Los resultados obtenidos en el caso de suelo flotante se muestran en la figura 10. Se comparan los resultados obtenidos con los valores dados por la norma. En la figura 11 se muestra el ajuste $a \mathrm{~K}_{\mathrm{ij}}=\mathrm{a}+\mathrm{b}$ log $f$ siguiendo el mismo razonamiento que en las uniones en cruz. Del mismo modo, el coeficiente b es cercano a 20.

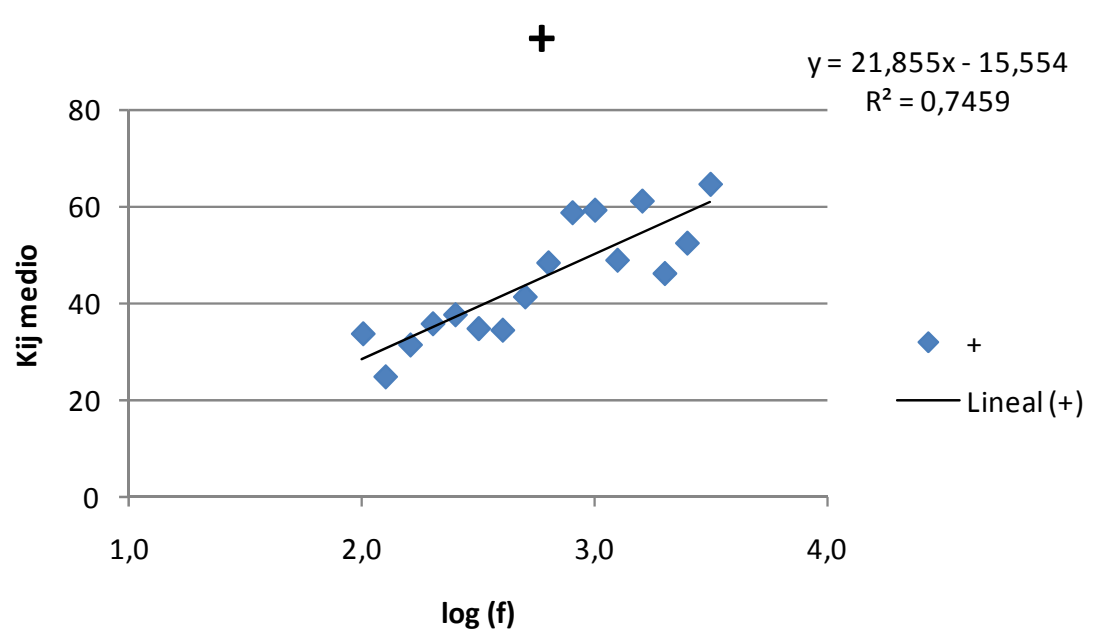

Fig. 8: Ajuste de los valores de $\mathrm{K}_{\mathrm{ij}}$ obtenidos para suelo flotante 
K12R.M=0. Rigido

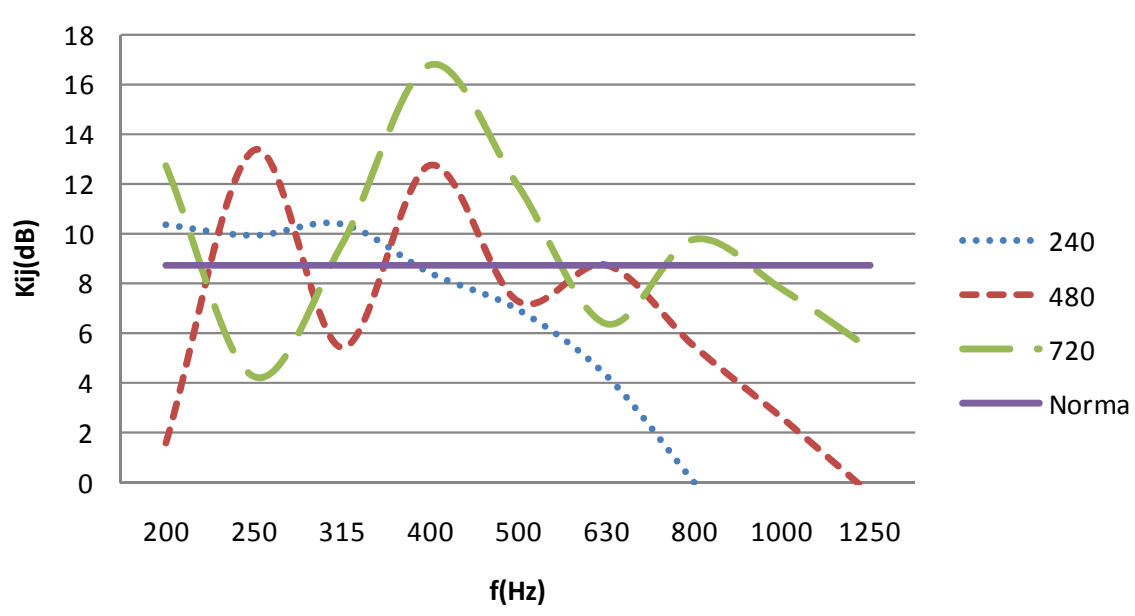

Fig. 9: Valores del índice de reducción vibracional K12R en unión en T.

\section{CONCLUSIONES}

La aplicación de la técnica de los elementos finitos ha permitido simular de forma sencilla las condiciones de ensayo del índice de reducción vibracional. Además se han podido comprobar limitaciones de los procedimientos de medida de forma más eficiente que siguiendo tales protocolos in situ. Esto se observa en la figura 5 donde se puede comprobar que, aun excitando a distancias permitidas por la normativa $(10848,2007)$ es más restrictivo situarnos cerca de la unión entre elementos que cerca de los límites de los mismos elementos. En el primer caso se supera el límite establecido por la expresión (3) con mayor dificultad.

En el caso de uniones rígidas se puede ver cómo los datos presentan ciertas oscilaciones en función de la frecuencia, aunque siguen una cierta tendencia horizontal. Estas oscilaciones también se presentan en los trabajos de Craik y Osipov (1995), Hopkins (1999), Crispin et al., (2006) y Alba et al. ( 2008), estudiando situaciones in situ. Por lo tanto, mediante la simulación por elementos finitos parece que nos acerquemos más a la realidad del comportamiento acústico de un conjunto constructivo que al considerar las predicciones empíricas de la normativa (CEN 12354, 2000).

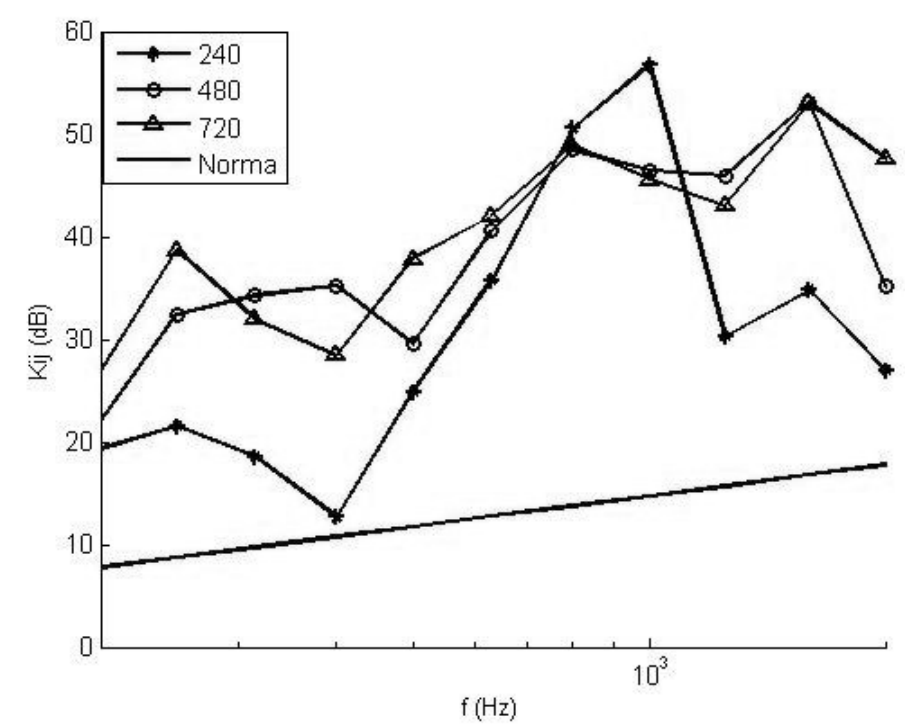

Fig. 10: K12R para suelo flotante y $M=0$, en unión en $\mathrm{T}$. 


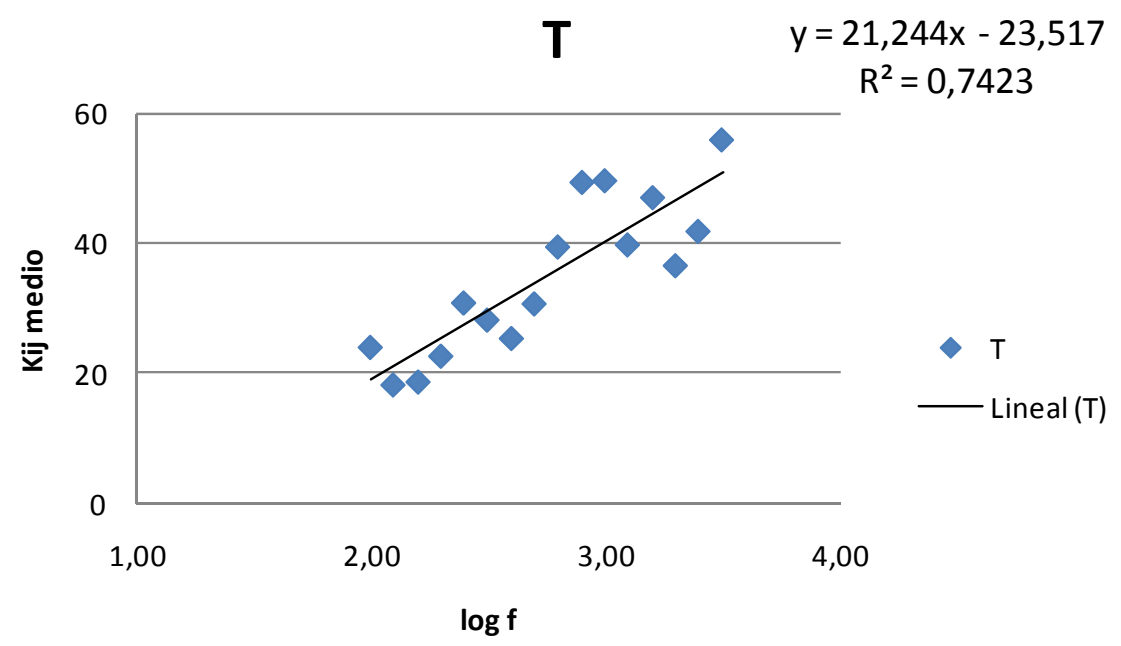

Fig. 11: Ajuste de los valores de Kij en suelo flotante con Unión en T.

Al introducir el suelo flotante se ha podido observar cómo la tendencia en función de la frecuencia era creciente en las simulaciones. Aunque las normas CEN-EN 12354-1 y CEN EN 12354-2 indican que se utilice los valores de las uniones rígidas, las simulaciones evidencian un claro comportamiento parecido al caso de uniones con elementos flexibles insertados. Esto se comprueba aún más cuando al realizar los ajustes aparece una tendencia frente al log(f) similar a la que se ofrecen en estas uniones. De esta manera se ha evidenciado que el considerar el conjunto formado por el suelo flotante como un elemento rígido, incluso excluyendo la masa del elemento flexible para el cálculo del índice de reducción vibracional (CEN 12354, 2000), se aleja de la realidad.

Por último, gracias a las simulaciones, se puede reajustar la formulación del parámetro $\Delta$ para el caso de otras relaciones entre el módulo de Young y espesor de la lámina elástica, propias del material, o reajustar la frecuencia de diseño del suelo flotante, en función de la carga sobre la lámina, tal y como se ha mostrado en las figuras 8 y 11.

\section{AGRADECIMIENTOS}

Este trabajo ha sido financiado por el Ministerio de Educación y Ciencia de España (BIA2007-68098C02-01 y BIA2007-68098-C02-02).

\section{REFERENCIAS}

Alba, J., Ramis J., Escuder, E. y Berto L. Airborne Sound Insulation of Hollow Brickwork, Building Acoustics 14 (3), 259-267 (2007)

Alba, J., Ramis, J., Escuder, E., del Rey, R. Effect of the estimation of the wall/floor junction type on the acoustic isolation. Acoustics'08. Paris. 29 Junio-4 Julio (2008)

ANSYS, Inc. www.ansys.com. Southpointe 275 Technology Drive Canonsburg, PA 15317

CEN EN 12354-1: Building Acoustics - Estimation of Acoustic Performance of Buildings from the Performance of Elements - Part 1: Airborne Sound Insulation Between Rooms (2000)

CEN EN 12354-2: Building Acoustics - Estimation of Acoustic Performance of Buildings from the Performance of Elements - Part 2: Impact Sound Insulation Between Rooms (2000)

Craik, R.J.M. y Osipov, A.G. Structural isolation of walls using elastic interlayers, Applied Acoustics 46, 233-249 (1995)

Craik R.J.M The contribution of long flanking paths to sound transmission in buildings, Applied Acoustics 62, 29-46 (2001). 
Crispin, C., Ingelaere, B., Van Damme, M., y Wuyts, D., The Vibration Reduction Index Kij: Laboratory Measurements for Rigid Junctions and for Junctions With Flexible Interlayers, Building Acoustics, 13 (2), 99-111(2006)

DB-HR CTE: Ley Española. REAL DECRETO 1371/2007, de 19 de octubre, por el que se aprueba el documento básico «DB-HR Protección frente al ruido» del Código Técnico de la Edificación (2007)

Hopkins, C., Measurement of the Vibration Reduction Index, Kij on Free-Standing Masonry Wall Constructions, Building Acoustics, 6 (3-4), 235-257 (1999)

ISO 10848: ISO 10848-parts 1, 2 and 3. Laboratory measurement of the flanking transmission of airborne and impact sound between adjoining rooms (2007)

Pedersen D. B. Estimation of Vibration Attenuation through Junctions of Building Structutes. Applied Acoustics 46, 285-305 (1995)

UNE EN ISO 3382-2:2008.Acústica. Medición de parámetros acústicos en recintos. Parte 2: Tiempo de Reverberación en recintos ordinarios (2008)

Zienkiewick O. C., Taylor R. L., El método de los elementos finitos, 4a edición Mc Grau Hill, CIMNE (Barcelona) Volumen 1 y 2 (1993) 
